“ C 2019 IEEE. Personal use of this material is permitted. Permission from IEEE must be obtained for all other uses, in any current or future media, including

reprinting/republishing this material for advertising or promotional purposes, creating new collective works, for resale or redistribution to servers or lists, or reuse of any copyrighted component of this work in other works." 


\title{
Beam Steering Conformal Transmitarray Employing Ultra-Thin Triple-Layer Slot Elements
}

\author{
Pei-Yuan Qin, Member, IEEE, Li-Zhao Song, and Y. Jay Guo, Fellow, IEEE
}

\begin{abstract}
A novel conformal transmitarray with beam steering ability is presented. First, an ultra-thin transmitarray element consisting of three layers of identical square ring slots is developed. The element has a thickness of $0.508 \mathrm{~mm}(0.04$ wavelength in the free space at $25 \mathrm{GHz}$ ), achieving a transmission phase range of $330^{\circ}$ with a maximum $3.6 \mathrm{~dB}$ loss. The element is then applied to a curved transmitarray conformal to a cylindrical surface fed by a standard gain horn with about a 10-dBi gain. A prototype is fabricated radiating a boresight beam with a peak measured gain of $19.6 \mathrm{dBi}$ and an aperture efficiency of $25.1 \%$. Second, when the transmitting surface of the above array is divided into two parts from the middle with different main beam directions, the combined beam can be radiated to an oblique angle with respect to the boresight direction. Using this method, a mechanical beam scanning conformal transmitarray antenna is designed. Its size is about 2.5 times larger than the fixed-beam one and consists of six transmitting surfaces with main beams directed to different angles. By rotating the feed horn to different positions, the main beam of the array can be switched to $\pm 15^{\circ}, \pm 10^{\circ}, \pm 5^{\circ}$ and $0^{\circ}$. A prototype is fabricated with a stable gain of about $18.7 \mathrm{dBi}$ at all beam angles.
\end{abstract}

Index Terms - Transmitarrays, beam steering, conformal array antennas.

\section{INTRODUCTION}

$\mathrm{T}$ ransmitarray antennas have attracted considerable attention due to their merits of high gain, low profile and flexible radiation performance [1-4]. Usually, they consist of multiple layers of flat antenna elements and an illuminating feed source. The phase of these elements are individually designed in order to provide appropriate phase responses to transform the spherical phase front from the feed into a planar phase front. As a result, a highly directive radiation beam can be realized with a high gain.

In the last decade, there have been substantial advances in transmitarray antennas. One of the most popular approach for transmitarray design is to use antenna elements based on multiple-layer frequency selective surfaces (FSS), which are multiple layers of printed radiators separated by dielectric materials or air gaps [5-11]. In order to meet the phase

Manscript received December 10, 2018; revised March 4, 2019; accepted April 26, 2019. This work was supported by the Australia Research Council Discovery Program under Grant DE170101203 and Grant DP160102219. (Corresponding author: Pei-Yuan Qin)

The authors are with the Global Big Data Technologies Centre (GBDTC), University of Technology Sydney (UTS), Ultimo, NSW 2007, Australia. compensation requirement, the elements are expected to cover a $360^{\circ}$ phase range with an acceptable transmission loss. It should be noted that it is very difficult to achieve this goal by using only one or two layers of FSS, and a multiple-layer FSS structure is required. It is reported that at least three layers of FSS are needed to accomplish sufficient phase shift range [1]. Employing this technology, various antennas elements are developed for transmitarray antennas, including a three-layer spiral dipole element [5], a four-layer double hexagonal ring elements [6], three-layer orthogonal dipoles [8], and multiplelayer subwavelength true time-delay elements [9-10]. Unfortunately, one potential drawback of this technology is the relatively high-profile of the antenna element, which is usually more than $0.5 \lambda_{0}$ ( $\lambda_{0}$ is the wavelength in the free space. Efforts have been devoted to reduce the profile of the transmitarray elements while still achieving the required $360^{\circ}$ phase range. In [11], a three-layer bandpass FSS element is presented that has a profile of $0.36 \lambda_{0}$. Another three-layer element with a thickness of $0.22 \lambda_{0}$ is demonstrated that consists of a split circular ring connected by a narrow strip in the middle layer and two polarizers in the upper and bottom layer [7]. In [12], a two-layer element is shown consisting of modified Malta crosses printed on a dielectric substrate with four vertical-plated through vias. The thickness of the element is $0.43 \lambda_{0}$. A two-layer element with two coplanar patches printed on the two sides of a printed circuit board (PCB) is proposed in [13] that can achieve a $180^{\circ}$ phase range. The patches are connected by a few through vias. The thickness of the element is $0.203 \mathrm{~mm}$ which is $0.05 \lambda_{0}$ at $77 \mathrm{GHz}$. It is worth pointing out that all the elements mentioned above are designed for flat transmitting surfaces, making them suitable for flat transmitarray antennas.

For many wireless communication platforms, such as satellites, aircrafts, and unmanned aerial vehicles (UAV), conformal high-gain antennas are needed in order to meet the aerodynamic requirements. For these applications, conformal transmitarray antennas, which are designed to follow the shapes of various mounting platforms, are highly desired as part of the platform surface, front in particular, can accommodate the transmitarray with a feed placed behind. Unfortunately, most of the current reported array elements are not suitable for conformal transmitarray antennas. As discussed above, they either consist of multiple thick layers of FSS or need to place vias between the top and bottom layers of elements. Therefore, it is very challenging to make these 
structures bent to conform to curved surfaces.

Another important requirement for transmitarray antennas is to scan the beams. Such beam scanning may not need complicated feed networks, thereby reducing the cost and losses especially at millimeter-wave or higher frequencies [14]. One typical approach to achieve electronically beam scanning for flat transmitarray antenna is to tune the transmission phase of the antenna element by using PIN didoes [15-17] and varactor didoes [18-19] to achieve discrete and continuous beam steering, respectively. For conformal transmitarray antenna, however, if the curvature is very large, it would be very challenging to integrate active elements on to the surface. One potential solution is to steer the beam by mechanically rotating the feed horn. Compared to electronic beam scanning, this solution can avoid the extra losses and costs associated with the usage of a very large number of active elements and complicated control circuits.

In light of the above literature review and discussion, it is found that there is very few work reported that achieved conformal transmitarray antennas and their beam steering. In this paper, we first report an ultra-thin transmitarray element that is suitable for conformal arrays. The element has three layers of square ring slots. Each layer is separated by a distance of only $0.02 \lambda_{0}$ at $25 \mathrm{GHz}$. Therefore, the entire thickness of the element is about $0.04 \lambda_{0}(0.508 \mathrm{~mm})$, making it suitable for bending. Although the element is very thin, it can achieve a transmission phase range of $330^{\circ}$ with a maximum $3.6 \mathrm{~dB}$ loss. Second, a transmitarray prototype using the proposed element that conforms to a cylindrical surface is designed, fabricated and measured. Third, we developed a new method to steer the beam of the proposed transmitarray. Instead of simply rotating the entire transmitting surface and the feed source together, only the feed source is rotated at the feed point. A prototype is designed and measured that can steer the beam to $0^{\circ}, \pm 5^{\circ}$, $\pm 10^{\circ}$ and $\pm 15^{\circ}$. Good agreement between simulated and measured results is achieved. To the authors' best knowledge, this is the first work ever reported on conformal transmitarray antennas with beam steering.

The rest of the paper is organized as follows. In section II, the overall design of the conformal transmitarray antenna is presented, including the element design, simulated and measured results of the transmitarray prototype. In Section III, the study on the beam steering of the proposed conformal transmitarray antenna is described followed by a discussion in Section IV. The paper concludes in SectionV.

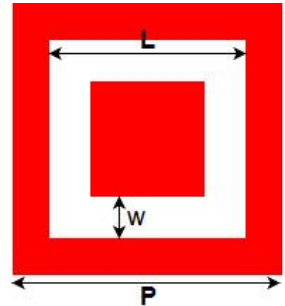

(a) Top view

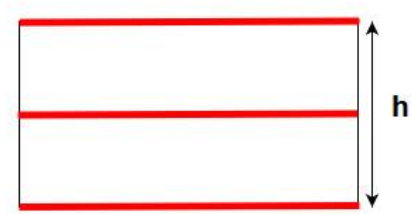

(b) Side view
Fig. 1. Top and side views of the proposed FSS element (Red part is the metal and white part is the substrate)

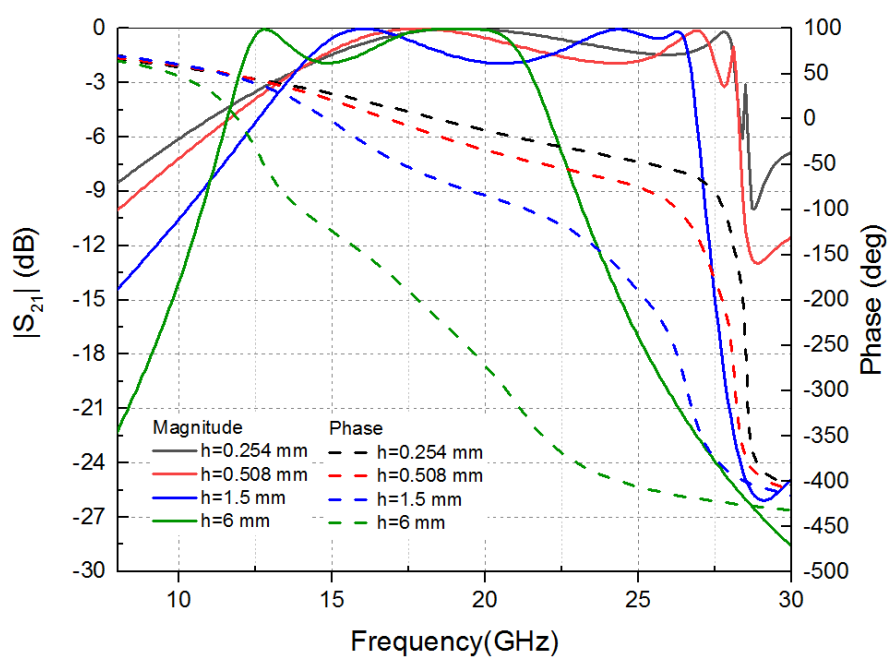

Fig. 2. Transmission performance of the planar transmitarray element with $L$ equals to $5 \mathrm{~mm}$ for different $h$ values.

\section{CONFORMAL TRANSMITARRAY DESIGN}

\section{A. Element Design}

The transmitarray element used in this work consists of three layers of identical square ring slots as shown in Fig. 1. The width and the length of the slot are $w$ and $L$, respectively. The unit periodicity is $P$ and the whole unit height is $h . w$ is chosen to be $0.8 \mathrm{~mm}$ and $P$ is $5.6 \mathrm{~mm}$. The transmission phase of the element can be varied with $L$ for a range of $3.5 \mathrm{~mm}$ to 5.41 $\mathrm{mm}$. The three-layer slot element employed has been reported in [20] for a planar multiple-polarization transmitarray. However, the total thickness of the element in [20] is $30 \mathrm{~mm}$, which is more than half of the wavelength at its operating frequency. In order to utilize this element for a conformal design, a parametric study of the effect of the height $h$ on the transmission coefficient is studied, which is given in Fig. 2. The element is simulated using the Floquet method with master-slave boundaries of 3D electromagnetic (EM) simulation software HFSS. When $h$ is varied, the other parameters are unchanged. It can be seen from Fig. 2 that the operating frequency goes higher when $h$ is reduced and the phase range is reduced with $h$. When $h$ equals to $6 \mathrm{~mm}$ which is $0.5 \lambda_{0}$ at $25 \mathrm{GHz}$, the phase range can cover more than $360^{\circ}$. However, for a $0.254 \mathrm{~mm}$ thickness, which is ideal for conformal design, the phase range is decreased significantly. Therefore, a compromise should be made between the 
thickness and the phase range. It is found that the PCB boards, which have a thickness of more than $1 \mathrm{~mm}$, will be easily broken when it is bent. Therefore, in this work, $h$ is chosen to be $0.508 \mathrm{~mm}$ which is about $0.04 \lambda_{0}$ at $25 \mathrm{GHz}$.

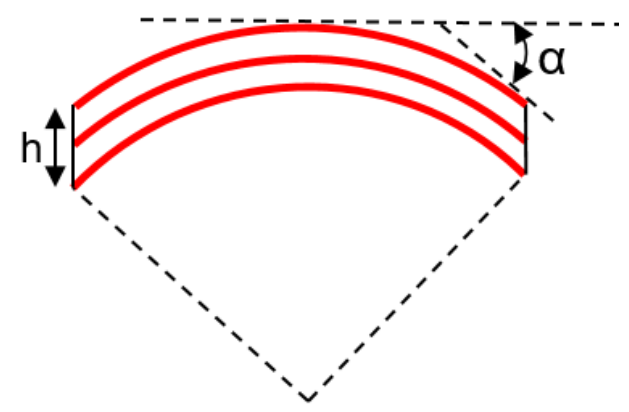

(a)

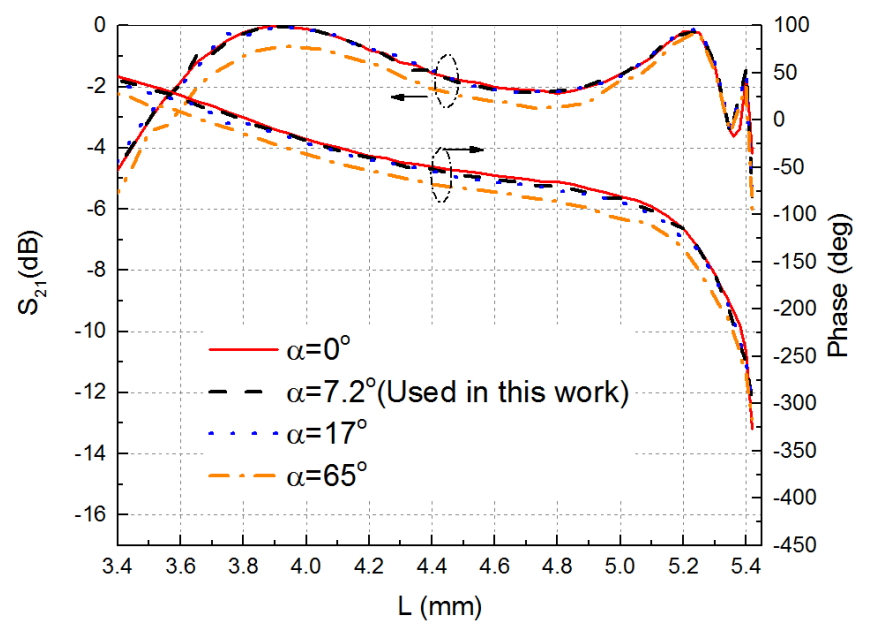

(b)

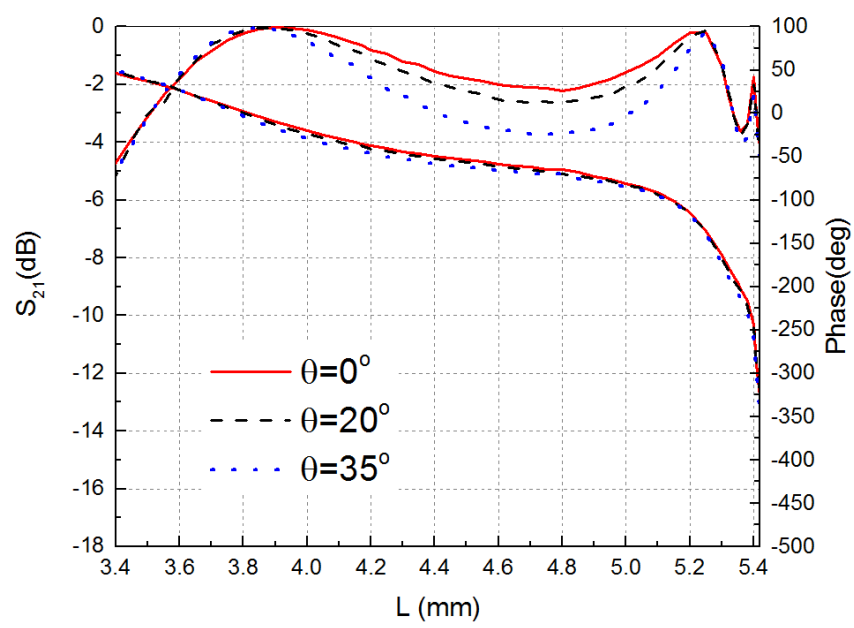

(c)

Fig. 3 Transmitarray element on a curved surface: (a) the element with a bending angle $\alpha$; (b) Simulated transmission amplitude and phase; (c)

Transmission performance of the curved element with $\alpha$ equal to $7.2^{\circ}$ at 25 $\mathrm{GHz}$ for normal and oblique incidence;
The above analysis is for a planar triple-layer square ring slot element. As the element is designed for conformal transmitarrays, its transmission coefficient is examined when it is bent with different curvature. As seen in Fig. 3 (a), the three-layer element is bent with different angles $\alpha$. When $\alpha$ is equal to zero degree, the element becomes a flat one. For the conformal transmitarray in this work, the element is bent with $\alpha$ equal to $7.2^{\circ}$. Fig. 3 (b) depicts the amplitude and phase of transmission coefficient versus slot length $L$. When $L$ varies from $3.5 \mathrm{~mm} \sim 5.41 \mathrm{~mm}$, a transmission phase range of $330^{\circ}$ is achieved for the flat element. The transmission loss $\left|\mathbf{S}_{21}\right|$ for most of the points are lower than $3 \mathrm{~dB}$. The worst case is 3.6 $\mathrm{dB}$ when $L$ is equal to $5.36 \mathrm{~mm}$. For the bent one with small values of $\alpha$, the phase range and the loss remain almost the same as the flat one. Only when $\alpha$ is very large, i.e. $60^{\circ}$, the transmission loss increases slightly, but it is still lower than $3.6 \mathrm{~dB}$. It is interesting to find that the phase range is nearly unchanged even for this large angle. This is because the phase change is determined by the length of the slot which is not affected by the curvature of the surface. It should be pointed out that the periodic boundary condition used in the simulation can only mimic an infinitely large plane surface rather than a cylindrical surface. Therefore, there may exist some discrepancy of the element performance between these two surfaces. However, as the bending angle of this work $\left(7.2^{\circ}\right)$ is not that large, the above simulation results can be used as a reasonably accurate reference when calculating the phases of the elements.

The transmission phase of the element is also examined for different incident angles as shown in Fig. 3 (c). Here, the oblique incidence is examined for the element bent with $\alpha$ equal to $7.2^{\circ}$. It can be seen that the losses increase with the angle of incidence for $L$ between $4 \mathrm{~mm}$ to $5.2 \mathrm{~mm}$. When the angle is equal to $35^{\circ}$ which is the largest one in this work, the maximum loss increases to $4 \mathrm{~dB}$ when $L$ is equal to $5.36 \mathrm{~mm}$. In terms of the phase range, it is almost unchanged for different incident angels. All in all, considering the ultralow profile, the $330^{\circ}$ phase range and a maximum $4 \mathrm{~dB}$ loss at the $35^{\circ}$ incidence angle achieved by the element is acceptable for conformal transmitarray antennas.

\section{B. Transmitarray Design}

As known, the transmitting phase of each array element on the surface should be designed to compensate for the spatial phase delay from the feed horn to that element, so that a certain phase distribution can be achieved to focus the beam at a specific direction [21]. Fig. 4 (a) and (b) show the 3D structure and the top view of the conformal transmitarray antenna with a prime focus, respectively. 


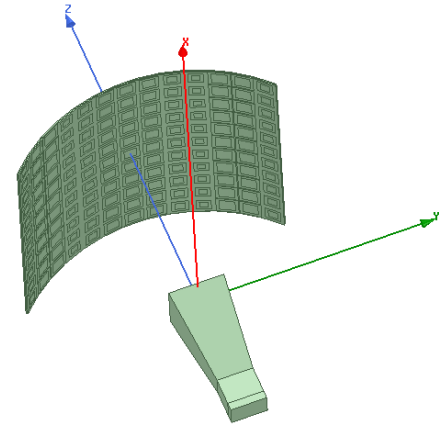

(a)

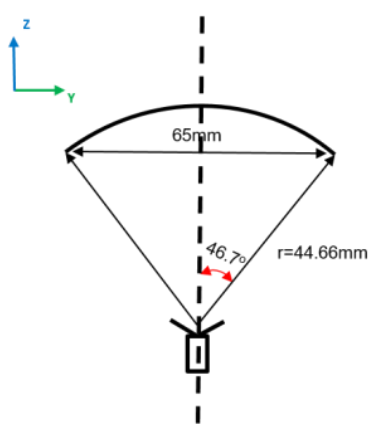

(b)
Fig. 4 (a) 3D structure of the conformal transmitarray; (b) Top view of the conformal transmitarray.

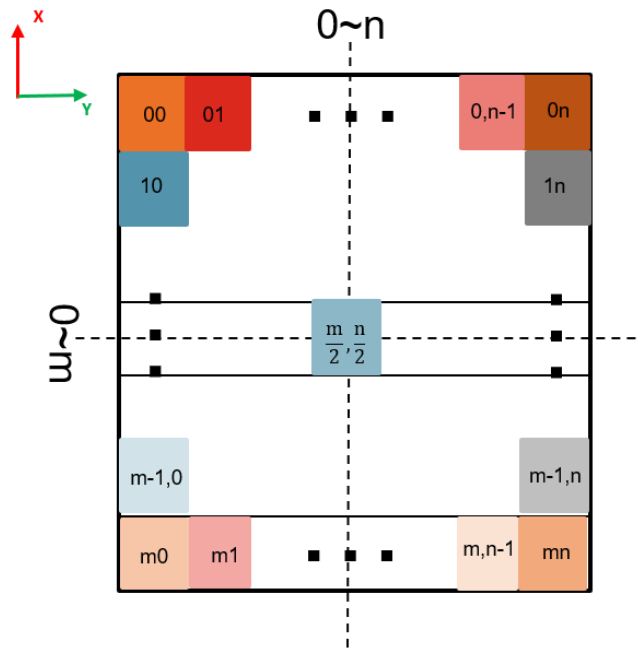

Fig. 5 Phase distribution of the unfolded conformal transmitarray

The feed horn is located in line with the middle of the cylindrical transmitarray surface. The unfolded transmitting surface is displayed in Fig. 5. Different colors represent units with different transmission phases. The phase $\varphi_{m n}$ for any unit is given in the following equation (1)

$$
\begin{gathered}
\varphi_{m n}=\varphi_{m, \frac{n}{2}}+r\left(\cos \theta_{r}-\cos \left(\theta_{n}-\theta_{r}\right)\right) * \frac{2 \pi}{\lambda_{0}} \\
=\varphi_{\frac{m}{2}, \frac{n}{2}}+r\left(\frac{1}{\cos \theta_{m}}+\cos \theta_{r}-\cos \left(\theta_{n}-\theta_{r}\right)-\tan \theta_{m} * \sin \varphi_{r}-1\right) * \\
\frac{2 \pi}{\lambda_{0}}
\end{gathered}
$$

where $\theta_{r}$ and $\varphi_{r}$ are radiation angles at yoz and xoz plane respectively, $\theta_{n}$ and $\theta_{m}$ are the half subtended angles of the cylindrical surface at yoz and $x o z$ plane, respectively, $\varphi_{m / 2, n / 2}$ is the transmission phase of the center unit, and $r$ is the distance from feed point to the center unit, which is chosen as $44.66 \mathrm{~mm}$ in this work.
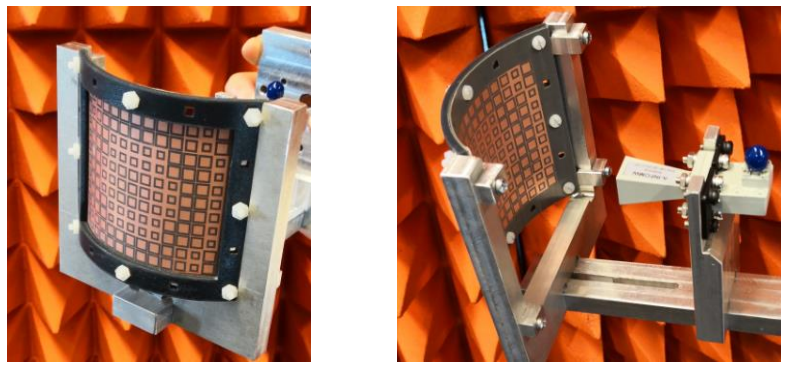

Fig. 6 Photograph of the conformal transmittarray prototype.

\section{Simulated and Measured Results}

A conformal transmitarray antenna prototype using the proposed element was designed. It consists of $13 \times 11=143$ elements. The cross section size of the transmitting surface is $65 \mathrm{~mm} \times 61.6 \mathrm{~mm}$. A standard gain horn LB-28-10-C-KF from A-INFO is used as the feed source and placed in the center of the cylindrical surface. The aperture edge illumination is around $-10 \mathrm{~dB}$ with a half subtended angle of $46.7^{\circ}$ for the conformal transmitting surface (as shown in Fig. 4(b)). Each layer of the unfolded transmitting surface was fabricated using standard PCB technology on low-cost Wangling F4B substrates (Dielectric Constant 2.2, $\tan \delta=$ 0.007). Then the two surfaces are laminated together and are attached to a 3-dimentional (3D) printed cylindrical frame. The whole array is mounted on a frame for antenna measurement purpose. The photograph of the prototype is shown in Fig. 6.

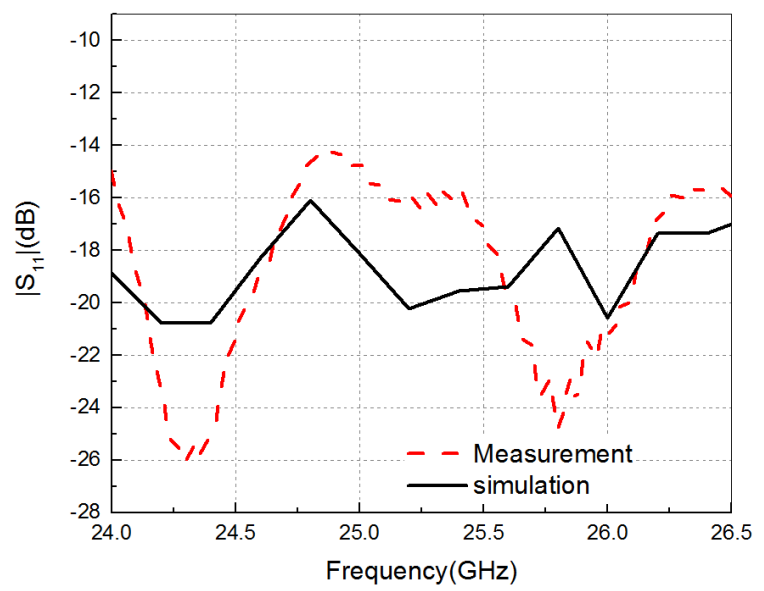

Fig. 7 Simulated and measured input reflection coefficients versus frequency of the antenna.

Fig. 7 shows the simulated and measured input reflection coefficients versus frequency of the antenna. As can be seen, the input reflection coefficient is below $-10 \mathrm{~dB}$ across the frequency band from $24 \mathrm{GHz}$ to $26.5 \mathrm{GHz}$. Reasonably good agreement between the simulated and measured results is achieved. Far-field radiation patterns were measured using a Microwave Vision Group (MVG) compact range antenna measurement system located at University of Technology Sydney, Ultimo, Australia. 


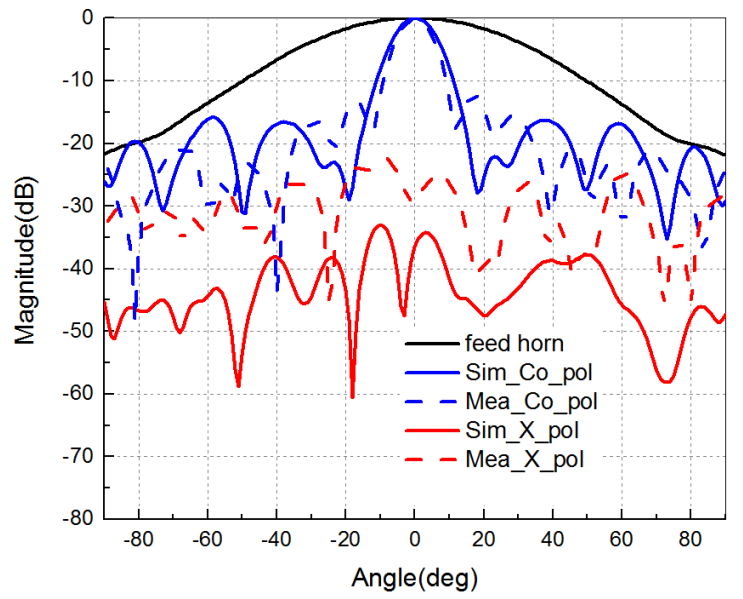

(a)

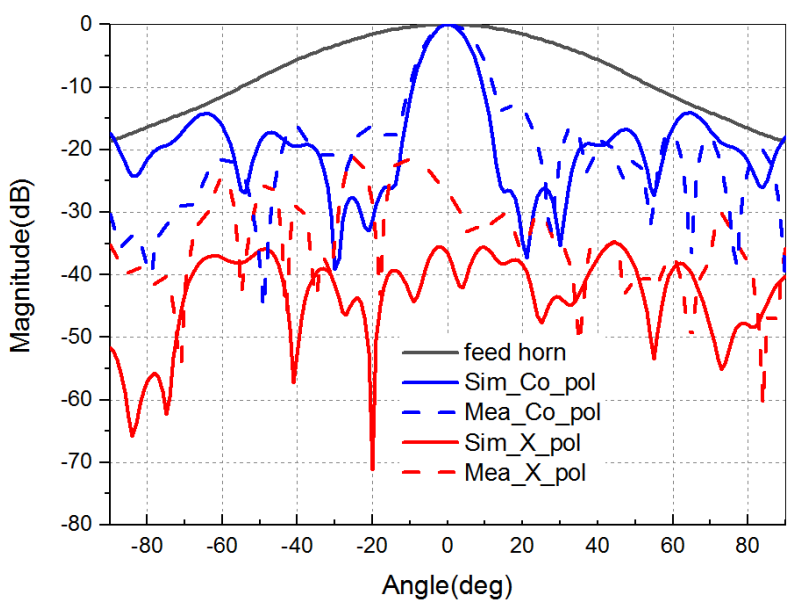

(b)

Fig. 8 Simulated and measured radiation patterns at $25.5 \mathrm{GHz}$ for: (a) E plane; (b) H-plane.

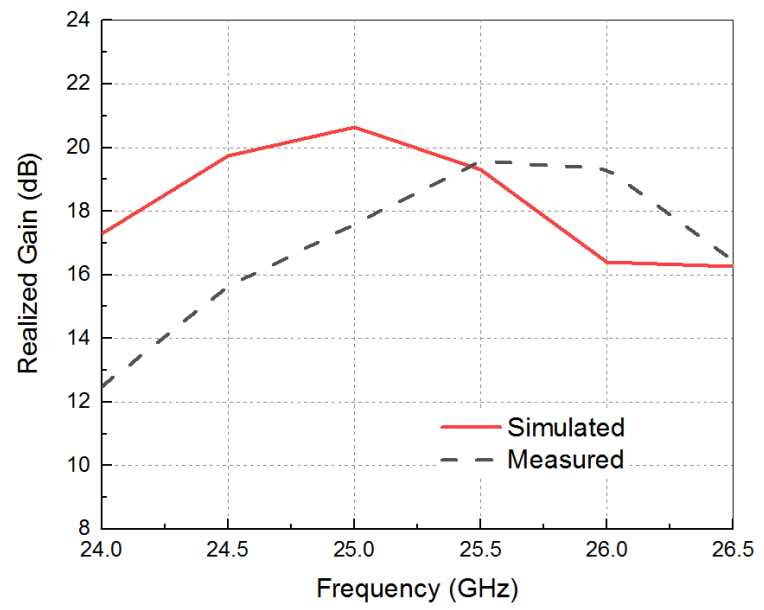

Fig. 9 Simulated and measured realized gains of the conformal transmitarray.

Fig. 8 shows the simulated and measured E- and H- plane patterns at $25.5 \mathrm{GHz}$, respectively. Compared to the feed horn antenna that has a gain of $10.6 \mathrm{dBi}$, the $3-\mathrm{dB}$ beam width of the proposed antenna is reduced from $54^{\circ}$ to $12^{\circ}$ for both planes. The measured cross-polarization level is less than -20
dB. The simulated and measured realized gains versus frequency are also found and plotted in Fig.9. The simulated peak gain is $20.5 \mathrm{dBi}$ at $25 \mathrm{GHz}$, while the measured one is $19.6 \mathrm{dBi}$ at $25.5 \mathrm{GHz}$. The measured 3-dB gain bandwidth is $6.7 \%$. The measured aperture efficiency is $25.1 \%$ considering the gain calculated by the aperture cross section size. The low efficiency and the small gain bandwidth are due to the use of very thin unit elements of the conformal transmitarray. As can be seen from Fig. 2, the array element's phase curve is sharper when the thickness is smaller. This leads to large phase correcting errors which may reduce the realized gains of the transmitarray and limit the gain bandwidth [1]. It is found that the efficiency and the gain bandwidth of the transmitarray can be increased to around $43 \%$ and $17 \%$, respectively, when the total thickness of the element is increased to $2.5 \mathrm{~mm}$. These simulation results are omitted here due to the limited space. The 0.508-mm-thick element selected in this work is for the ease of bending for conformal transmitarrays to verify the design concept. In principle, a conformal transmitarray using 2.5-mm-thick elements could be fabricated using direct ink printing technology, but this would be far beyond our budget and our access to the technology.

There are some discrepancies in the input reflection coefficients, $2 \%$ frequency shift and around $1 \mathrm{~dB}$ gain difference of the simulated and measured peak gains. They can be mostly attributed to following factors. First, there can be some inaccuracies in the fabrication process and alignment errors. Second, the PCB board used to fabricate the antenna is a low-cost one, which could have a varied dielectric constant value and a varied loss tangent value from the data sheet.

\section{MeChanically ReConfigurable Conformal TRANSMITARRAY DESIGN}

For the transmitarray discussed in last section, it can only radiate a beam at a fixed direction. Electronic beam scanning can be achieved by employing active components, such as PIN diodes or varactor diodes, to change the transmission phase of the array elements according to the beam direction required [15-18]. Usually, electronically reconfigurable transmitarray will involve a large number of active elements as the phase of each element needs to be changed independently. This may lead to extra loss, cost and control networks. Furthermore, for conformal transmitarray design, if the curvature is very large, it may not be easy to integrate active elements on a curved surface.

This section demonstrates a method to achieve a mechanical beam scanning of the conformal transmitarray reported in Section II. The method employed is to rotate the feed source only rather than simply rotating the entire transmitting surface and the feed source together. Actually, for many conformal applications, the transmitting surface, which could be the skin of communication platforms, cannot be easily moved.

\section{A. Scanning Mechanism}

For a non-reconfigurable single transmitarray, each array element on the transmitting surface is designed to radiate the 
beam towards a specific angle, e.g. $0^{\circ}$. In order to achieve the beam scanning by rotating the feed source, the transmitting surface reported in Section II is divided into two parts from the center line, as shown in Fig.10 (a). If one part of the transmitting surface is designed to direct the beam to $\varphi 1$, and the other part is for $\varphi 2$, the combined beam is directed to $(\varphi 1+$ $\varphi 2) / 2$. It should be pointed out that in order not to have a split beam, the two beams cannot be separated far away and be too narrow. In this work, it is found that a step of about $10^{\circ}$ serves as an optimum value based on simulated results.

The transmitting surface proposed in this work is shown in Fig. 10 (b), consisting of six parts marked as $a 1, a 2, a 3, b 1$, $b 2, b 3$. They are designed to radiate in different beam directions with respect to the $z$ axis, as shown in Fig. 11 .

The subtended angles of the parts $a 1, a 2, b 1, b 2$ are $36^{\circ}$ and they are $18^{\circ}$ for $a 3$ and $b 3$. Therefore, the total subtended angle of the transmitting surface is $180^{\circ}$, making the surface a half circle. The feed gain horn LB-28-15-C-KF from A-INFO is placed in the center with $12.2 \mathrm{~dB}$ gain at $25 \mathrm{GHz}$. For each operating state of the transmitarray, a transmitting surface with a $72^{\circ}$ subtended angle is illuminated by the standard gain horn, resulting in a $-10 \mathrm{~dB}$ edge illumination. In this case, the adjacent transmitarray surface parts will have minimum effects on the active surface. The transmitarray element size is the same as that reported in Section II. Each $72^{\circ}$ transmitting surface consists of $15 \times 13$ elements. The cross section size is $78.6 \mathrm{~mm} \times 72.8 \mathrm{~mm}$.

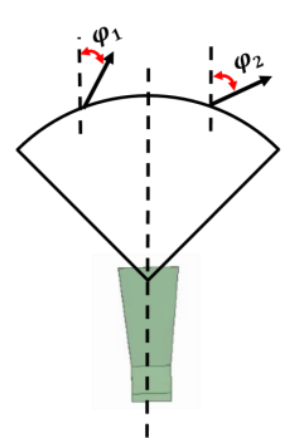

(a)

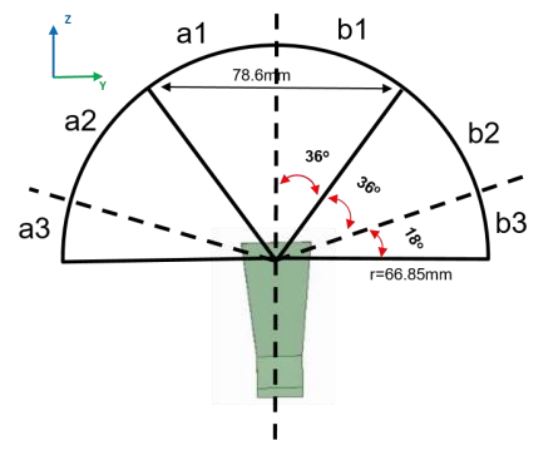

(b)
Fig. 10 (a) Passive conformal transmitarray in Section II; (b) Reconfigurable conformal transmitarray.

When the feed horn is pointed to the center, the center two parts of the surface is illuminated as indicated with red stripe line shown in Fig. 11 (a). As the two parts direct the beams to $-5^{\circ}$ and $5^{\circ}$, respectively, the combined beam is towards $0^{\circ}$. If the horn is rotated anti-clockwisely by $18^{\circ}$, the active surface would change as shown in Fig. 11 (b). In this case, three parts are illuminated, namely, a1, half of a2, and half of b1. Then the combined beam will be directed to $\left(\varphi_{\mathrm{a} 1}+\left(\varphi_{\mathrm{a} 2} / 2+\varphi_{\mathrm{b} 1} / 2\right)\right) / 2$ $=\left(-5^{\circ}+\left(-15^{\circ} / 2+5^{\circ} / 2\right)\right) / 2=-5^{\circ}$

Using the same principle, when the feed horn continues to rotate by another $18^{\circ}$, as shown in Fig. 11 (c), transmitting surface $a l$ and $a 2$ will be active and the beam will be directed to $-10^{\circ}$. The beam will be directed to $-15^{\circ}$ when the feed is rotated by a further $18^{\circ}$ as shown in Fig. 11 (d). When the feed horn is rotated clockwisely, the main beam of the antenna can be directed to $5^{\circ}, 10^{\circ}, 15^{\circ}$, respectively, due to the structural symmetry.

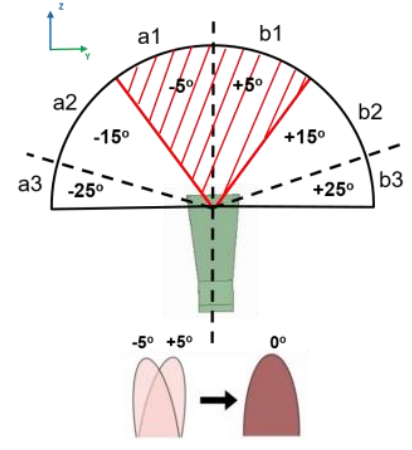

(a)

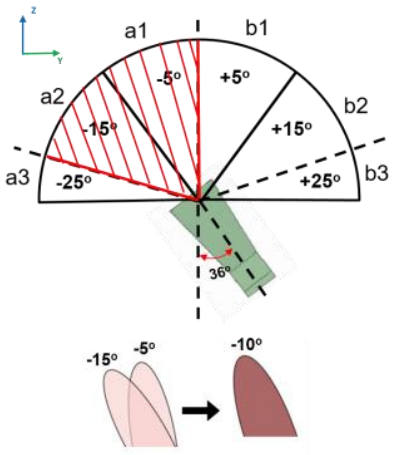

(c)

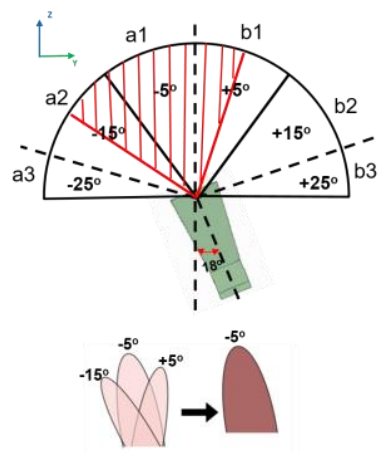

(b)

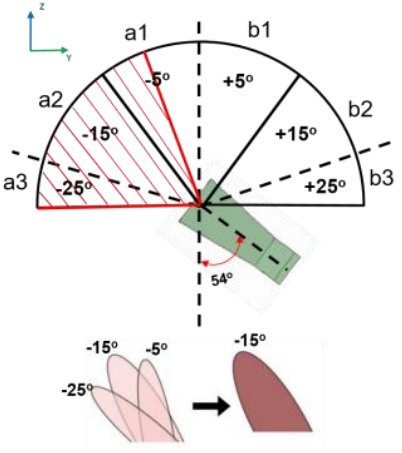

(d)
Fig. 11 Different operating states of the conformal transmitarray corresponding to different beam directions: (a) $0^{\circ}$; (b) $-5^{\circ}$; (c) $-10^{\circ}$; (d) $-15^{\circ}$.
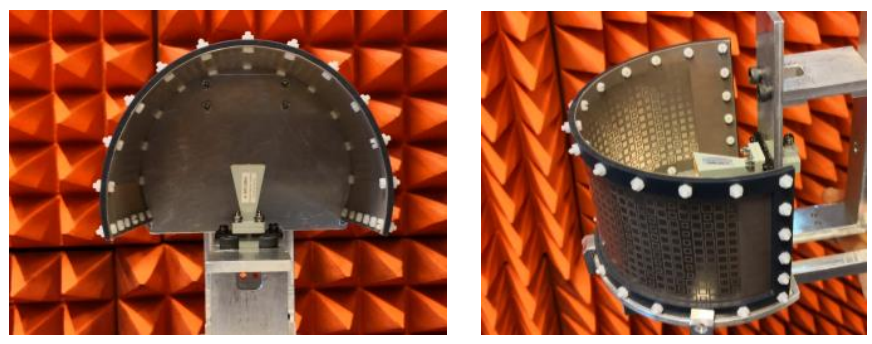

Fig. 12 Photograph of the reconfigurable transmittarray prototype.

\section{B. Experimental Results}

To verify the design concept discussed above, a reconfigurable conformal transmitarray prototype working at $25 \mathrm{GHz}$ is designed and fabricated. The array is fabricated using Rogers DiClad 880 substrate (Dielectric Constant 2.2, $\tan \delta=0.001)$ using the same method as the fixed-beam conformal transmitarray in Section II. The two-layer surface is mounted on a platform with a rotatable gain horn. An angle square is printed at the back of the platform so that the gain horn can be rotated to any required angle. Photograph of the prototype is shown in Fig.12. 


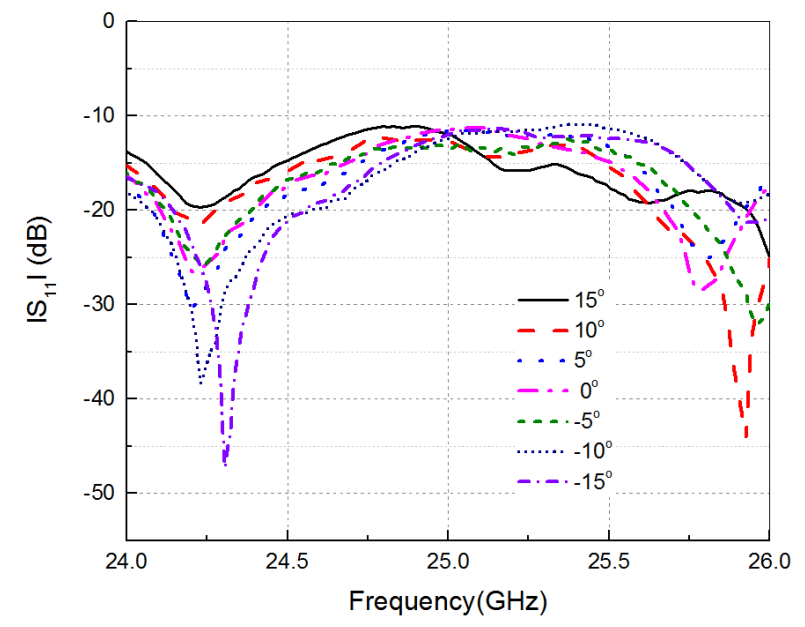

Fig. 13 Measured input reflection coefficients of the reconfigurable conformal transmitarray.

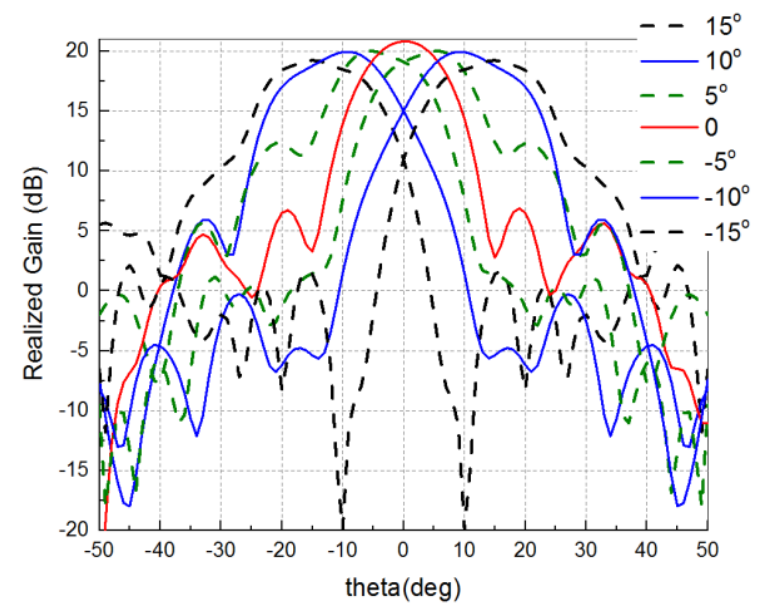

(a)

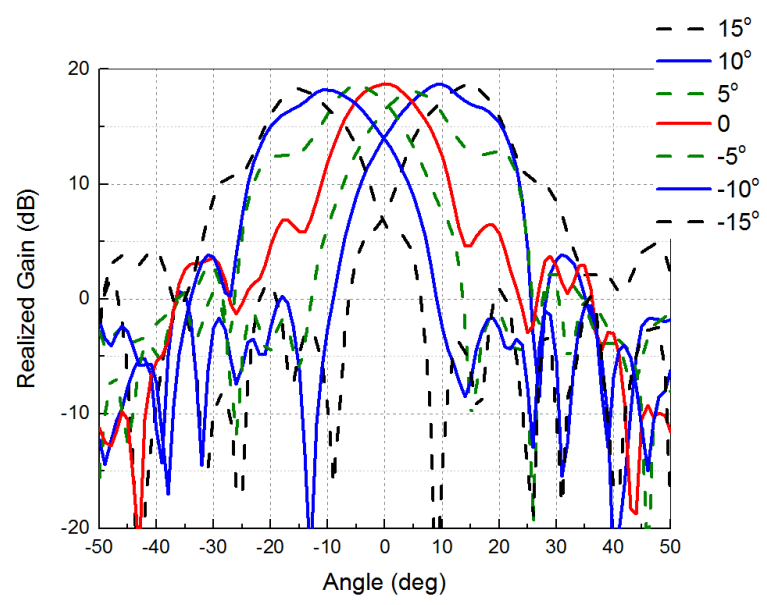

(b)

Fig. $14 \mathrm{H}$-plane radition patterns at $25 \mathrm{GHz}$ for different states of the transmitarray: (a) Simulated resutls; (b) Measured resutls.

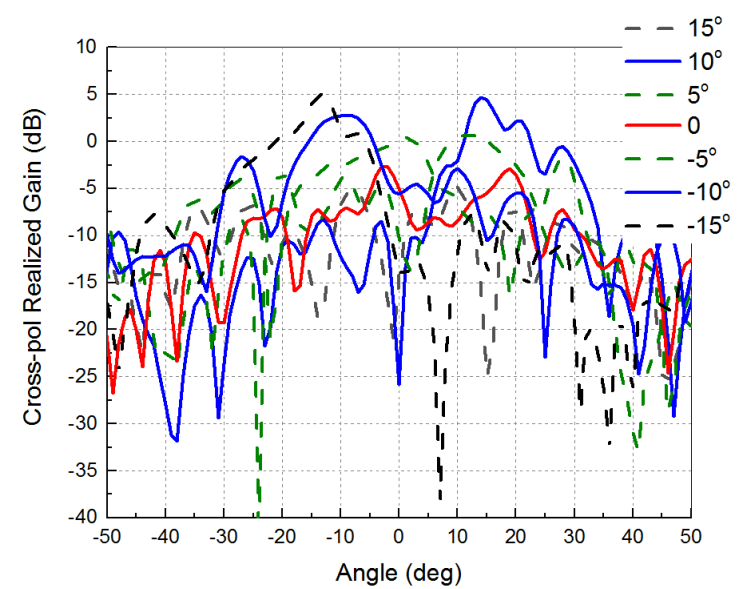

Fig. 15 Measured cross-polarization level in H-plane at $25 \mathrm{GHz}$ for different states of the transmitarray.

The input reflection coefficients and the radiation patterns were measured for seven different positions of the gain horn. It is seen from Fig. 13 that the measured input reflection coefficients are below $-10 \mathrm{~dB}$ for all the seven states from 24 $\mathrm{GHz}$ to $26 \mathrm{GHz}$. Measured and simulated gain curves in $\mathrm{H}$ plane are compared at $25 \mathrm{GHz}$ in Fig. 14. The simulated gains at boresight is $20.3 \mathrm{dBi}$ and around $19.5 \mathrm{dBi}$ for other scanning angles. While for measured results, a stable gain value of about $18.7 \mathrm{dBi}$ is achieved at all angles. Therefore, the scan loss of this mechanically reconfigurable conformal transmitarray is very small. As the beam in E plane is not changed, the patterns are omitted here. They are similar to those of the fixed-beam transmitarray given in Fig. 8. The absolute values of cross-polarization at $25 \mathrm{GHz}$ are given in Fig. 15. The maximum cross polarization is $5 \mathrm{dBi}$ for $15^{\circ}$ scanning, which makes the relative cross polarization level lower than $-14 \mathrm{~dB}$. For each working state with a $72^{\circ}$ transmitting surface, the simulated aperture efficiency is $17.8 \%$, while the measured one is $14.8 \%$. The efficiency of the scanned transmitarray is lower than that of the fixed one. This is mainly due to the beam combination used to generate the steered beam. As a result, the realized gain of the scanned array is lower than that of the fixed one.

\section{DISCUSSION}

This section discusses the beam scanning range of the developed conformal transmitarray. As discussed in the last section, each main beam of the array is the combination of the beams of two or three parts of the transmitting surface. Therefore, the beam scanning range is related to the beam direction of each part $\theta_{r n}$ (with respect to the $z$ axis), the subtended angle of each part $\theta_{h}$ and the number of the parts $2 n$ ( $n$ for each half of the transmitting surface). In this design, $\theta_{h}$ is chosen as $36^{\circ}$ to satisfy $-10 \mathrm{~dB}$ edge illumination. As a result, the maximum value of $n$ is $180^{\circ} / 36^{\circ}=5$. The beam directions for the parts $a l$ and $b 1$ are chosen to be $-5^{\circ}$ and $5^{\circ}$, respectively, resulting in a $10^{\circ}$ step. If this step is increased, the combined beam will tend to be split and the gain will drop. If the step is decreased, the total scanning range will be 
reduced. Therefore, the $10^{\circ}$ step serves as an optimum tradeoff value based on a few simulated results. Following this angle step, we have the parts $a 2$ and $a 3$ radiating to $-15^{\circ}$ and $-25^{\circ}$, respectively. Note that $-25^{\circ}\left(\theta_{r_{-} a 3}\right)$ is with respect to the $z$ axis. If considering the center axis of the part $a 3$ (which is $-y$ axis), it is $65^{\circ}$ as shown in Fig. 16. As is known, the directivity will drop significantly when the beam is scanning away too much from the direction perpendicular to the transmitting surface. For this reason, the beam combined from $a 2$ and $a 3$, which points to $-20^{\circ}$, has a reduced gain and is not used in this array. As a result, only half of $a 3$ is used in the array for a $-15^{\circ}$ beam direction (as seen from Fig.11 (d)). Therefore, the subtended angles of $a 3$ and $b 3$ are half of the other parts. Also, there is no need to include more parts for larger beam angles as the gain will be reduced further. One possible method to increase the beam scanning range is to reduce the subtended angel of each transmitting surface part so that more surface parts can be included. This would be studied in our future work.

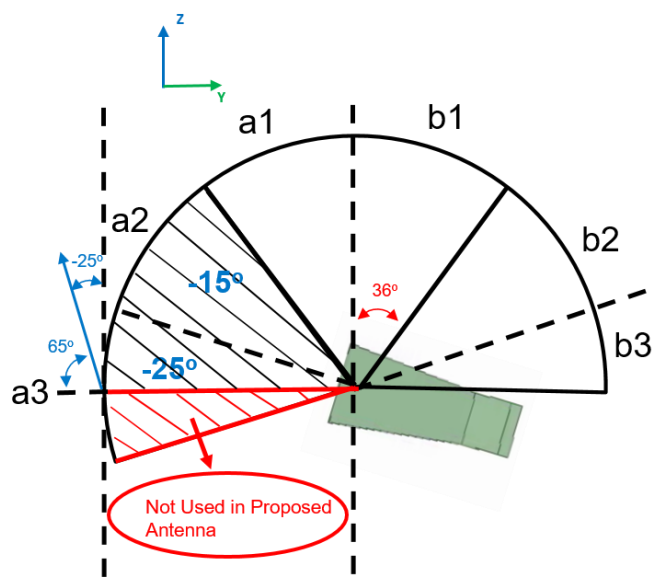

Fig. 16 Beam steering range limit of the proposed conformal tranmsitarray antenna.

Comparing to a fixed-beam conformal transmitarray consisting two parts, for example, $a l$ and $b l$, the total size of the reconfigurable one is only about 2.5 times larger, but, seven beam directions can be achieved. For some communication platforms where the size of the surface is not a primary constraint, the reported beam scanning method can be used.

It should be noted a straightforward way to achieve beam scanning is to employ several identical transmitting surfaces and rotate the feed horn to the center of each surface, as shown in Fig. 17. But the number of the beam directions is dependent on the number of the transmitarray antennas used and the angle step is usually large. For example, if three identical transmitting surfaces consisting of $a l$ and $b l$ are used as shown in Fig. 17, three beams pointing at $-72^{\circ}, 0^{\circ}$, and $72^{\circ}$ are achieved when the feed horn is switched to the center of each surface.

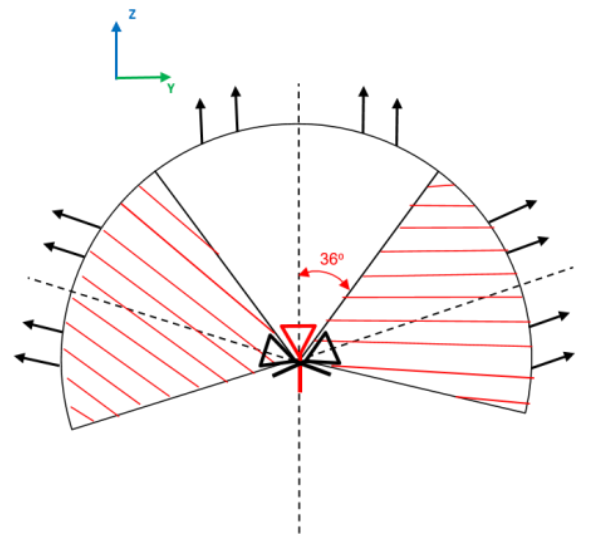

Fig. 17 Three transmitarray antennas for three beam directions, $-72^{\circ}, 0^{\circ}$, and $72^{\circ}$.

\section{V.CONCLUSION}

A conformal transmitarray and its beam steering is presented in this paper. First, an ultra-thin array element is developed, consisting of three-layer of identical square ring slots. The total thickness of the element is $0.508 \mathrm{~mm}$ which is $0.04 \lambda_{0}$ at $25 \mathrm{GHz}$. A transmission phase range of $330^{\circ}$ is realized with a maximum $3.6 \mathrm{~dB}$ loss. Second, a curved transmitarray that is conformal to a cylindrical structure is designed using the element. A peak measured gain of $19.6 \mathrm{dBi}$ at $25.5 \mathrm{GHz}$ is obtained. Third, by combing transmitting surfaces with different beam directions and rotating the feed horn, beam steering of a conformal transmitarray is realized. The size of the reconfigurable transmitarray is approximately 2.5 times of the array with the fixed beam, while it can switch the beam to $\pm 15^{\circ}, \pm 10^{\circ}, \pm 5^{\circ}$ and $0^{\circ}$ with a stable realized gain of $18.7 \mathrm{dBi}$. Measured results agree well with simulated ones. The developed transmitarray and its beam steering one may find wide applications in communication systems where conformal structure is needed.

\section{REFERENCES}

[1] A. H. Abdelrahman, F. Yang, A. Z. Elsherbeni, P. Nayeri, C. A. Balanis, Analysis and Design of Transmitarray Antennas, San Francisco, CA, USA: Morgan \& Claypool, 2017.

[2] Q. Cheng, H. F. Ma, and T. J. Cui, "Broadband planar Luneburg lens based on complementary metamaterials," Applied Physics Letter, vol. 95, no. 18, 2009.

[3] S.-W. Qu, H. Yi, B. J. Chen, K. B. Ng, and C. H. Chan, "Terahertz reflecting and transmitting metasurfaces," Proc. IEEE, vol. 105, no. 6, pp. 1166-1184, Jun. 2017.

[4] Z.-W. Miao, Z.-C. Hao, G. Luo, "140 GHz high-gain LTCC-Integrated transmit-array antenna using a wideband SIW aperture-coupling phase delay structure," IEEE Trans. Antennas Propag., vol. 66, no. 1, pp. 182190, Jan. 2018.

[5] A. H. Abdelrahman, A. Z. Elsherbeni, and F. Yang, "High gain and broadband transmitarray antenna using triple-layer spiral dipole elements," IEEE Antennas Wireless Propag. Lett., vol. 13, pp. 12881291, 2014.

[6] P.-Y. Feng, S.-W. Qu, S. Yang, L. Shen, and J. Zhao, "Ku-band transmitarrays with improved feed mechanism," IEEE Trans. Antennas Propag., vol. 66, no. 6, pp. 2883-2891, Feb. 2018.

[7] P.-Y. Feng, S.-W. Qu, and S. Yang, "Octave bandwidth transmitarrays with a flat gain," IEEE Trans. Antennas Propag., vol. 66, no. 10, pp. 5231-5238, Oct. 2018. 
[8] A. Aziz , F. Yang, S. Xu, and M. Li, "An efficient dual-band orthogonally polarized transmitarray design using three-dipole elements," IEEE Antennas Wireless Propag. Lett., vol. 17, no. 2, pp. 319-322, Feb. 2018.

[9] M. Li and N. Behdad, "Wideband true-time-delay microwave lenses based on metallo-dielectric and all-dielectric lowpass frequency selective surfaces," IEEE Trans. Antennas Propag., vol. 61, no. 8, pp. 4109-4119, Aug. 2013.

[10] M. Li, M. A. Al-Joumayly, and N. Behdad, "Broadband true-time-delay microwave lenses based on miniaturized element frequency selective surfaces," IEEE Trans. Antennas Propag., vol. 61, no. 3, pp. 1166-1179, Mar. 2013.

[11] Q. Luo, S. Gao, M. Sobhy, and X. Yang, "Wideband transmitarray with reduced profile," IEEE Antennas Wireless Propag. Lett., vol. 17, no. 3, pp. 450-453, Mar. 2018

[12] W. An, S. Xu, F. Yang, and M. Li, "A double-layer transmitarray antenna using malta crosses with vias," IEEE Trans. Antennas Propag., vol. 64, no. 3, pp. 1120-1125, Mar. 2016.

[13] S. B. Yeap, X. Qing, and Z. N. Chen, "77-GHz dual-Layer transmitarray for automotive radar applications," IEEE Trans. Antennas Propag., vol. 63, no. 6, pp. 2833-2837, Jun. 2015.

[14] S. V. Hum and J. Perruisseau-Carrier, "Reconfigurable reflectarrays and array lens for dynamic antenna beam control: a review," IEEE Trans. Antennas Propag., vol. 62, no. 1, pp. 183-198, Jan. 2014.

[15] L. Palma, A. Clemente, L. Dussopt, R. Sauleau, P. Potier, and P. Pouliguen, "Circularly-polarized reconfigurable transmitarray in KaBand with beam scanning and polarization switching capabilities," IEEE Trans. Antennas Propag., vol. 65, no. 2, pp. 529-540, Feb. 2017.
[16] C. Huang, W. Pan, X. Luo, "Low-loss circularly polarized transmitarray for beam steering application," IEEE Trans. Antennas Propag., vol. 64, no. 16, pp. 4471-4476, Oct. 2016.

[17] M. Wang, S. Xu, F. Yang, and M. Li, "Design of a Ku-band 1-bit reconfigurable transmitarray with $16 \times 16$ slot coupled elements," 2017 IEEE AP-S, San Diego, CA, USA.

[18] J. Y. Lau and S. V. Hum, "A wideband reconfigurable transmitarray element," IEEE Trans. Antennas Propag., vol. 60, no. 3, pp. 1303-1311, 2012.

[19] J. G. Nicholls, and S. V. Hum, "Full-space electronic beam-steering transmitarray with integrated leak-wave feed," IEEE Trans. Antennas Propag., vol. 64, no. 8, pp. 3410-3422, 2016.

[20] X. Zhong, L. Chen, Y. Shi, and X. Shi, "Design of multiple-polarization transmitarray antenna using rectangle ring slot element," IEEE Antennas Wireless Propag. Lett., vol. 15, pp. 1803-1806, 2016.

[21] J. Huang and J. A. Encinar, Reflectarray Antennas, by Institute of Electrical and Electronics Engineers, John Wiley \& Sons, 2008. 